\title{
Acute bronchiolitis in a paediatric emergency department of Northern Greece. Comparisons between two decades
}

$\overline{\text { Afroditi Sakellaropoulou' } 1 \text { Maria Emporiadou', Victor Aivazis², John Mauromixalis }{ }^{1}, \text { Maria Hatzistilianou }^{1}}$

12nd Paediatric Department, Aristotle University of Thessaloniki, AHEPA Hospital, Greece 21st Paediatric Department, Aristotle University of Thessaloniki, AHEPA Hospital, Greece

Submitted: 27 March 2011

Accepted: 2 January 2012

Arch Med Sci 2012; 8, 3: 509-514

DOI: 10.5114/aoms.2012.29279

Copyright (c) 2012 Termedia \& Banach

\section{Abstract}

Introduction: Acute bronchiolitis is the most common lower respiratory tract infection in infants and toddlers concerning small bronchi or bronchioli. This retrospective study aimed to evaluate the incidence of acute bronchiolitis and the use of $\beta_{2}$-agonists between two different decades.

Material and methods: During 1990-1991 and 2001-2002, the files of the $2^{\text {nd }}$ Paediatric Emergency Department of Aristotle University of Thessaloniki, AHEPA Hospital were reviewed and cases of acute bronchiolitis were recorded and analysed.

Results: During 1990-1991, 14538 children were identified with respiratory infections and bronchiolitis was diagnosed in 519/14 538 children (3.56\%). Only 34 out of 519 patients received nebulised salbutamol (6.6\%) and 221/519 were hospitalized (42.6\%). During 2001-2002, 9001 children were found to have respiratory tract infections and acute bronchiolitis was diagnosed in 641/9001 of them (7.12\%). In total, 411/641 children (64.1\%) received salbutamol and ipratropium, and 89/641 patients $(13.88 \%)$ were hospitalized. There was a predominance of male sex in both decades $(p=0.509)$. There was a statistically significant difference $(p<0.001)$ concerning the use of nebulised salbutamol with nebulised ipratropium between the two decades. Finally, during 2001-2002, the use of bronchodilators with or without corticosteroids was more frequent and it appears to be correlated with the reduced number of admissions to hospital $(p<0.05)$ compared with 1990-1991. Conclusions: There was an increase in the incidence of acute bronchiolitis during the last decade. The admission rate decreased probably due to the use of nebulized salbutamol and ipratropium, but further multicentre comparative trials are required to define the role of bronchodilators in the treatment of acute bronchiolitis.

Key words: acute bronchiolitis, management, respiratory infections.

\section{Introduction}

Acute bronchiolitis is the most common lower respiratory tract infection in infants and toddlers concerning infection of the small bronchi or bronchioli [1]. Bronchiolitis is a clinical diagnosis based on typical history and findings on physical examination [2]. It is considered as the first episode of wheezing and it is typically described in children younger than 2 years old [2].

There is no specific diagnostic test or gold standard that confirms the diagnosis or excludes other diseases that may be clinically similar (e.g.,

\section{Corresponding author:} Afroditi Sakellaropoulou MD $2^{\text {nd }}$ Paediatric Department Aristotle University of Thessaloniki AHEPA Hospital 18 Salaminos Brilissia 15235 Athens, Greece Phone: +306972881018 E-mail: wx@otenet.gr 
bacterial pneumonia) [2]. The clinical picture of bronchiolitis was provided by the American Academy of Pediatrics in its 2006 position statement, based on which children with bronchiolitis typically have "rhinitis, tachypnea, wheezing, cough, crackles, use of accessory muscles, and/or nasal flaring" [3]. However, since the definition of acute bronchiolitis remains descriptive and includes all infants with wheezing, debates exist between clinicians over whether an acute lower respiratory tract infection in young children with wheezing should be diagnosed as bronchiolitis, reactive airways disease, or even asthma [4].

Few studies exist concerning the epidemiology and the clinical course of bronchiolitis. Moreover, the differences in defining bronchiolitis can cause difficulties in comparing data between various studies. Despite the high prevalence of bronchiolitis, little consensus exists also on the optimal management of the disease. There is great variation in therapeutic practice among hospital paediatricians and private practitioners. Apart from the use of oxygen therapy and attention to fluid and nutritional status, the evidence base for all other therapies drops sharply. Nevertheless, the most common therapies used are bronchodilators, systemic and inhaled corticosteroids and antibiotics [5]. Therefore, national guidelines could prove helpful in reducing this disparity.

The purpose of this study was to compare the morbidity due to acute bronchiolitis between infants who visited the Paediatric Emergency Department (PED) during the years 1990-1991 and 2001-2002, to study and report their epidemiological and clinical features, to compare clinical practice guidelines and recommendations, and physician practice management of bronchiolitis at the PED.

\section{Material and methods}

This is a retrospective study that included all children who visited the Paediatric Emergency Department of the $2^{\text {nd }}$ University Paediatric Department of Aristotle University of Thessaloniki, at AHEPA Hospital, in the period from 1 January 1990 to 31 December 1991, and also from 1 January 2001 to 31 December 2002, with acute respiratory infections, and especially acute bronchiolitis.

For each child material was collected from the hospital medical files in order to fulfil the research protocol that included information about their personal history, symptoms of the disease and their management.

\section{Statistical analysis}

Data were analysed using the Statistical Package for the Social Sciences (SPSS) Version 11.2 (Chicago, IL). The quantitative variables were analysed with descriptive statistics, and qualitative variables were described with frequencies. The data were presented as mean \pm standard deviation (SD).

Comparison between categorical indicators was performed using the $\chi^{2}$ test and Fisher's exact test. Student's $t$-test and ANOVA were used when the studied parameters fulfilled the criteria of normal distribution and equal variations. Distributional properties of continuous data were used in order to evaluate whether the distributional assumptions were satisfied. Otherwise, non-parametric assays, such as Mann-Whitney test and Kruskal-Wallis test, were used.

The level of statistical significance was set at 0.05 and the confidence interval at $95 \%$.

\section{Results}

In the period 1990-1991, 18616 children were examined at the PED of the $2^{\text {nd }}$ Paediatric Department of Aristotle University of Thessaloniki, of whom 14538 came due to respiratory problems (78.09\%). In the 2-year period 2001-2, 19964 children visited the PED, and 9001 had respiratory problems (45.08\%).

During the years 1990-1991, acute bronchiolitis was diagnosed in 519/14538 children with respiratory tract infection (3.56\%) at the PED. The average age of children with acute bronchiolitis was $9.17 \pm 6.27$ months (range: 0.03-24 months). Three hundred and sixty-eight out of 519 infants with acute bronchiolitis were younger than 12 months (70.9\%), 149/519 of them were in the age group of $12-24$ months (28.7\%), and in 2 cases the exact age was not recorded (0.4\%). Three hundred and one out of 519 of them were boys (58\%) and 218/519 were girls (42\%) (Table I).

There was a statistically significant difference ( $p=0.019$ ) between the average age of boys (8.61 \pm 5.92 months) and that of girls with acute bronchiolitis (9.92 \pm 6.67 months).

Two hundred and twenty-one out of 519 patients with acute bronchiolitis were hospitalized (42.6\%) and there was a statistically significant difference between the mean age of hospitalized children (6.54 \pm 4.53 months) and non-hospitalized children (11.11 \pm 6.67 months) $(p<0.001)$.

Five patients with acute bronchiolitis (1\%) had an underlying disease (3 congenital cardiopathy, 1 laryngomalacia, and 1 bronchopulmonary dysplasia). There were 24 children (4.62\%) who had coexisting otitis and 2 patients also had laryngitis $(0.38 \%)$. Moreover, 3 of the 5 patients with an underlying disease were hospitalized ( $p=0.655$ ).

One hundred and seventy-nine out of 519 cases of acute bronchiolitis were recorded in spring (34.48\%), 163/519 in winter (31.4\%), 89/519 during summer (17.14\%) and 88/519 in autumn (16.95\%). Only $34 / 519$ patients (6.6\%) received nebulised short-acting $\beta_{2}$-agonist (salbutamol) and 1 patient received intramuscular methylprednisolone $(0.2 \%)$ (Table II). 
Table I. Demographics, co-morbidity and clinical findings in the two groups

\begin{tabular}{|c|c|c|c|}
\hline Demographics & Years 1990-1991 & Years 2001-2002 & Value of $p$ \\
\hline Age [months] & $9.17 \pm 6.27$ & $10.93 \pm 6.98$ & $<0.001$ \\
\hline Male/female [\%] & $301 / 218(58 \% / 42 \%)$ & $382 / 259(59.6 \% / 40.4 \%)$ & 0.509 \\
\hline Comorbidity [\%] & $5 / 519(1 \%)$ & $7 / 641(1.1 \%)$ & 1 \\
\hline Hospitalization & $221 / 519(42.6 \%)$ & $89 / 641(13.9 \%)$ & $<0.001$ \\
\hline \multicolumn{4}{|l|}{ Clinical findings } \\
\hline \multicolumn{4}{|l|}{ Physical findings: } \\
\hline - Fever & $49 / 519(9.4 \%)$ & $121 / 641(18.9 \%)$ & \\
\hline - Wheezing & $83 / 519(16 \%)$ & $141 / 641(22 \%)$ & \\
\hline - Rales [\%] & $84 / 519(16.2 \%)$ & $108 / 641(16.84 \%)$ & \\
\hline - Dyspnoea & $26 / 519(5 \%)$ & $6 / 641(0.9 \%)$ & \\
\hline \multicolumn{4}{|l|}{ Laboratory findings: } \\
\hline - WBC $[K / \mu l]$ & $10445.54 \pm 6054.30$ & $11951.57 \pm 4326.08$ & \\
\hline - CXR findings [\%]: & $117 / 519$ (22.54\%) & $64 / 641(9.98 \%)$ & \\
\hline - Atelectasis/infiltrate & 9/519 (1.7\%) & $13 / 641(2 \%)$ & \\
\hline - Hyperinflation & $45 / 519(8.7 \%)$ & $10 / 641(1.6 \%)$ & \\
\hline - Normal & $63 / 519(12.1 \%)$ & $41 / 641(6.4 \%)$ & \\
\hline
\end{tabular}

Table II. Management of acute bronchiolitis at the PED during the years 1990-1991 and 2001-2002

\begin{tabular}{|lcc|}
\hline Therapeutic management of acute bronchiolitis & $\begin{array}{c}\text { Patients during } \\
1990-1991 \\
n(\%)\end{array}$ & $\begin{array}{c}\text { Patients during } \\
2001-2002 \\
n(\%)\end{array}$ \\
\hline Nebulised $\beta_{2}$-agonist (salbutamol) & $0(0 \%)$ & $15 / 641(2.3 \%)$ \\
\hline Nebulised $\beta_{2}$-agonist (salbutamol) with nebulised ipratropium $p$ & $34 / 519(6.6 \%)$ & $236 / 641(36.8 \%)$ \\
\hline Nebulised $\beta_{2}$-agonist (salbutamol) with corticosteroids & $0(0 \%)$ & $5 / 641(0.78 \%)$ \\
\hline $\begin{array}{l}\text { Nebulised } \beta_{2} \text {-agonist (salbutamol) with nebulised ipratropium } \\
\text { and corticosteroids }\end{array}$ & $0(0 \%)$ & $151 / 641(23.55 \%)$ \\
\hline Corticosteroids & $1519(0.2 \%)$ & $7 / 641(0.156 \%)$ \\
\hline $\begin{array}{l}\text { Nebulised } \beta_{2} \text {-agonist (salbutamol) with nebulised ipratropium } \\
\text { and nebulised adrenaline }\end{array}$ & $0(0 \%)$ & $3 / 641(0.6 \%)$ \\
\hline $\begin{array}{l}\text { Nebulised } \beta_{2} \text {-agonist (salbutamol) with nebulised ipratropium, } \\
\text { corticosteroids and nebulised adrenaline }\end{array}$ & $0(0 \%)$ & $1 / 641(0.156 \%)$ \\
\hline Corticosteroids and nebulised adrenaline & $0(0 \%)$ & $1 / 641(0.156 \%)$ \\
\hline Nebulised adrenaline & $0(0 \%)$ & $1 / 641(0.156 \%)$ \\
\hline \begin{tabular}{l} 
No medical treatment \\
\hline
\end{tabular} & $484 / 519(93.25 \%)$ & $221 / 641(43.47 \%)$ \\
\hline
\end{tabular}

During the years 2001-2002, acute bronchiolitis was diagnosed in 641/9001 children (7.12\%) with respiratory tract infection who were examined at the PED. Their mean age was $10.94 \pm 6.981$ months (range: 1-24 months). Three hundred and eighty-one out of 641 children with acute bronchiolitis were younger than 12 months (59.4\%), 255/641 were in the age group $12-24$ months (39.8\%) and in 5 cases the exact age was not recorded (0.8\%). Three hundred and eighty-six children (60.2\%) were boys and 255 were girls (39.8\%).

The mean age of boys with acute bronchiolitis (10.95 \pm 7.04 months) did not differ significantly from that of girls (10.91 \pm 6.906 months) ( $p=0.948)$. Seven out of 641 patients (1.1\%) had an underlying disease (2 congenital cardiopathy, 1 congenital benign muscle hypotonia, 1 achondroplasia, 1 psycho- motor delay, 1 mental retardation and 1 hydrocephalus) and 3/7 patients (42.85\%) were hospitalized $(p=0.026)$.

Two hundred and twenty-four out of 641 cases were reported in spring (34.94\%), 222/641 during winter months (34.63\%), 140/641 in autumn (21.84\%) and 55/641 patients in the summer months (8.58\%). Eighty-nine out of 641 patients were hospitalised due to severe bronchiolitis (13.9\%). There was a statistically significant difference ( $p=0.005)$ between the mean age of hospitalised children ( $9 \pm 7.71$ months) and that of non-hospitalised patients (11.25 \pm 6.8 months).

Their treatment included nebulised $\beta_{2}$-agonist (salbutamol) alone (2.3\%) or in combination with nebulised ipratropium (36.8\%) or corticosteroids $(0.78 \%)$ or even combination with ipratropium and 
corticosteroids (23.55\%). Seven out of 641 children received only corticosteroids (1.09\%), 3/641 nebulised salbutamol with ipratropium and nebulised adrenaline $(0.46 \%)$, and $1 / 641$ nebulised salbutamol with ipratropium, nebulised adrenaline and corticosteroids $(0.156 \%)$. One out of 641 patient $(0.156 \%)$ received only nebulised adrenaline, while 221/641 patients did not receive any medical treatment (34.47\%) (Table II). In total, 411/641 children (64.1\%) received salbutamol with ipratropium with or without corticosteroids at the PED, and only a small percentage of them $(24 / 641,3.74 \%)$ were hospitalized $(p<0.001)$.

There was a predominance of male sex in both decades ( $p=0.509$ ), while during the years 1990-1991 younger infants with bronchiolitis visited the PED compared with 2001-2002 ( $p<0.001$ ) (Table I). Clinical and radiological findings of the patients are presented in Table I.

There was a statistically significant difference $(p<0.001)$ concerning the use of nebulised salbutamol with nebulised ipratropium between the two decades (Table II) and also the hospitalization rate was higher during 1990-1991 compared with the years 2001-2002 $(p<0.001)$ (Table I).

\section{Discussion}

Acute bronchiolitis is considered to be the most common viral infection of the lower airways in infants. Acute bronchiolitis occurs in epidemics in the winter months. In tropical and subtropical climates, these epidemics occur during the rainy season [6]. In our study, the majority of cases were observed during spring and winter (Figure 1).

Based on previous findings the risk of bronchiolitis is seasonal and is associated with male gender [7], as in our study, where the majority of patients were of male gender in both decades (Table I). Moreover, younger infants, including premature infants and infants younger than 6 weeks [7], are thought to be susceptible to viral infections as

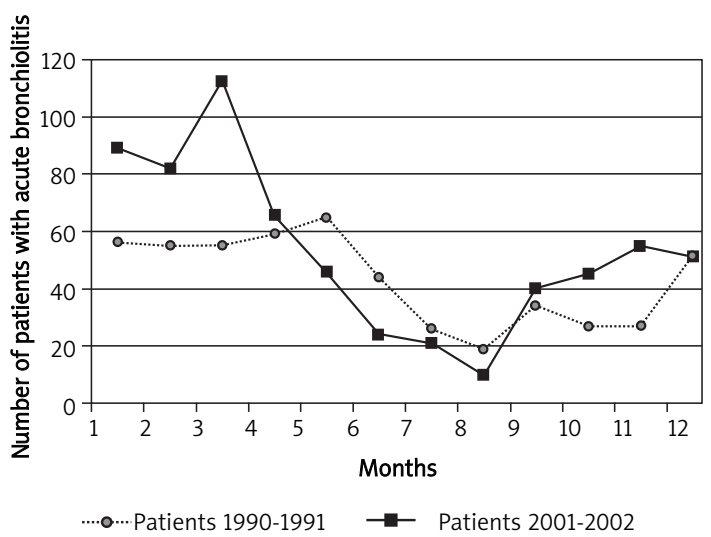

Figure I. Monthly distribution of patients with acute bronchiolitis during the years 1990-1991 and 2001-2002 a result of missing the transplacental transfer of antibodies [8]. Furthermore, those with co-existing cardiac or respiratory disease, and neonates 1-3 months old, are more prone to require admission to hospital [7, 8], as in our report, where during the years 1990-1991 and 2001-2002, 3/5 and 3/7 admitted patients respectively had an underlying disease, and hospitalized infants were younger than outpatients $(p<0.05)$. Therefore, the findings of our study suggest that the presence of an underlying disease and the young patient's age are risk factors for a severe degree of bronchiolitis and admission to the hospital. The mechanisms that contribute to increased disease severity in infants in each of these risk groups are not fully understood, but are probably related to abnormalities in physiological and immunological responses to infection [7].

The majority of children with bronchiolitis develop only a mild disease and can be treated at home [9]. In the US, during the years 1997-2000, more than 700000 visits to emergency departments were reported for infants with lower respiratory tract infections during the Respiratory Syncytial virus (RSV) period, and $29 \%$ of them were admitted to the hospital [10]. In our study, an increase of visits to the PED by infants with acute bronchiolitis (7.12\%) was recorded during the 2001-2002 period compared with 1990-1991 (3.56\%). Some of the possible reasons for the observed increase include overall trends in child care practice, changes in hospitalization practices, and the possibility of increased pathogen virulence. There may also be an increase in visits resulting from increased awareness of asthma and children's respiratory health in general or an unmeasured host factor that is causing more children to become symptomatic.

Most children have a self-limiting illness and are managed in the community although around 1-3\% of all infants require admission to hospital [11]. Hospitalisation with bronchiolitis peaks between 3 and 6 months of life [12]. Bronchiolitis associated hospitalizations have increased considerably since 1980 and have become a problem of public health worldwide [13]. The cause is likely to be multi-factorial, although it is possible that the use of pulse oximetry is related to the increased admission rates [11]. This finding is contrary to our findings, where reduction of admission rates was observed due to acute bronchiolitis from $42.6 \%$ to $3.74 \%$ between the two decades $(p<0.001)$, probably due to the way that cases of bronchiolitis were managed at the PED, and due to early sensitization of patients.

Another possible explanation of the reduction in the admission rates in cases with acute bronchiolitis between the two decades is the fact that there is a high diversity of the genotype of the responsible aetiological microorganisms for bronchiolitis, which could explain the observation that in 1 year the 
cases of acute bronchiolitis are milder compared with the next year. Therefore, the mean duration of hospitalization is shorter and also the need for supplemental oxygen is reduced, although the possibility of such an event is reduced with the collection of results from two continuous winters, as in our study [14].

Bronchodilators, epinephrine and corticosteroids have been used in the treatment of bronchiolitis. Apart from oxygen therapy [6], fluid therapy, aspiration of secretions and ventilation support, few treatment options will be beneficial. There are doubts about the efficacy of inhaled bronchodilators (salbutamol or adrenaline), with or without hypertonic saline solution, suggesting that these options should be selectively used as therapeutic trials in moderatesevere bronchiolitis [6].

Bronchodilators produce small short-term improvements in clinical scores. However, based on various studies no significant benefit of bronchodilator treatment was noted among infants hospitalized for bronchiolitis. Therefore, bronchodilators are not currently recommended for infants with bronchiolitis. They are also correlated with increased costs and increased adverse effects. Moreover, the rate and duration of hospitalization were not significantly reduced in the group treated with bronchodilators versus the control group [15].

A Cochrane review [16] of eight randomised trials in mild to moderate bronchiolitis (total of 394 children) showed that bronchodilator treatment was associated with a significant improvement in clinical scores. However, this comparison could have been biased, because some of these studies included patients with recurrent wheezing. The improvement in clinical scores was not regarded as clinically significant and there was no improvement in oxygenation or rates of hospital admission.

The American Academy of Pediatrics (AAP) recommends that "bronchodilators should not be used routinely in the management of bronchiolitis" [17]. Anticholinergic agents, such as ipratropium bromide, have been studied in multiple trials and their use appeared to be beneficial [18, 19]. In our study, during the years 2001-2002 increased incidence of the use of various medications at the PED has been recorded, and also various combinations of medicines. To be more precise, the use of bronchodilators (nebulised salbutamol with ipratropium) with or without corticosteroids was frequent, and it appears to be correlated with the reduced number of admissions to hospital $(p<0.05)$.

The use of bronchodilators to treat children with bronchiolitis remains controversial. The controversy may be the result of differences in study populations, in choice of bronchodilators, and in measured outcome variables between trials. Thus, the existing studies lead to confounding results concerning the use of epinephrine $[10,20,21]$. In our study, none of the patients received nebulised adrenaline during the years 1990-1991, while during the years 2001-2002, 4/641 patients only with acute bronchiolitis received nebulised adrenaline alone or in various combinations, which is a very small percentage in order to extract statistically significant conclusions.

Controversies also exist concerning the effect of corticosteroids on clinical symptoms, length of hospital stay (LOS) and duration of symptoms, compared with the placebo in hospitalized infants with bronchiolitis [20-25].

The Pediatric Emergency Care Applied Research Network [26] and the Pediatric Emergency Care Applied Research Network study [20] did not find any benefit from the use of corticosteroids for the treatment of bronchiolitis in hospital admission rates, clinical severity scores, length of stay for admitted patients, or adverse events for those treated with corticosteroids. Finally, the American Academy of Pediatrics (AAP) recommends that "corticosteroid medication should not be used routinely in the management of bronchiolitis" [17]. However, in our study, there was a negative correlation between the use of corticosteroids and admission to the hospital during the years 1990-1991 $(r=-0.038$ and $p=0.390)$ and 2001-2002 ( $r=-0.113$ and $p=0.004)$ respectively.

In our study, patients received a variety of medical treatments, especially during 2001-2002, whereas $151 / 641$ (23.55\%) received nebulised $\beta_{2}$-agonist (salbutamol) with nebulised ipratropium and corticosteroids, and very few were treated with corticosteroids alone or in various combinations, as can be seen in Table II. Moreover, it appears that during the years 2001-2002 there was an increased tendency of pharmacological treatment of acute bronchiolitis compared with the years 1990-1991 ( $p<0.001$ ).

One of the disadvantages of this study was that it did not evaluate the mode of rehydration therapy, such as nasogastric or intravenous, but only the pharmacological interventions, such as bronchodilators and corticosteroids. Moreover, this is not a controlled study, and our findings were dependent on retrospective data records. These findings also reflect physician practice at one point of time, but practice patterns constantly evolve, and this was mainly the practice of senior physicians. Finally, our study could not provide data on infectious versus non-infectious causes of bronchiolitis and on the final outcome of patients since it is a retrospective study based on recorded data from the outpatient department.

In conclusion, evaluating diagnostic tests for bronchiolitis is problematic because it is a disease that is diagnosed clinically. Thus, there is no gold standard against which to compare testing strategies. Moreover, despite the high prevalence of bronchiolitis, little consensus exists on optimal management. There is no specific treatment with a clear beneficial effect on the course of the disease. Based 
on the existing criteria, supportive care, including administration of oxygen and fluids, is the cornerstone of treatment of acute bronchiolitis. The role of bronchodilators in the treatment of bronchiolitis has been the subject of many studies and systematic evidence-based reviews of the literature. Even score-based studies are difficult to compare, because many of the measures used do not have established validity or proven correlation with clinically significant improvement. Therefore, bronchiolitis continues to be an active area of investigation and further studies are needed to explore the combination of these therapies and other interventions, such as nebulised hypertonic saline. Large placebocontrolled randomized controlled trials that utilize consistent and validated outcomes are needed to settle the question of the effect of bronchodilators and corticosteroids on acute bronchiolitis.

\section{References}

1. Barben J, Kuehni CE, Trachsel D, Hammer J. Management of acute bronchiolitis: can evidence based guidelines alter clinical practice? Thorax 2008; 63: 1103-9.

2. Bordley WC, Viswanathan M, King VJ, et al. Diagnosis and testing in bronchiolitis: a systematic review. Arch Pediatr Adolesc Med 2004; 158: 119-26.

3. American Academy of Pediatrics Subcommittee on Diagnosis and Management of Bronchiolitis. Diagnosis and management of bronchiolitis. Pediatrics 2006; 118: 1774-93.

4. Mansbach JM, Camargo CA Jr. Bronchiolitis: lingering questions about its definition and the potential role of vitamin D. Pediatrics 2008; 122: 177-9.

5. Menif K, Kechaou W, Bouziri A, et al. Current management of acute bronchiolitis in Tunisia. Tunis Med 2007; 85: 665-8.

6. Chan PW, Chew FT, Tan TN, Chua KB, Hooi PS. Seasonal variation in respiratory syncytial virus chest infection in the tropics. Pediatr Pulmonol 2002; 34: 47-51.

7. Smyth RL, Openshaw PJ. Bronchiolitis. Lancet 2006; 368: 312-22.

8. Koehoorn M, Karr CJ, Demers PA, Lencar C, Tamburic L, Brauer M. Descriptive epidemiological features of bronchiolitis in a population-based cohort. Pediatrics 2008; 122: 1196-203.

9. King VJ, Viswanathan M, Bordley WC, et al. Pharmacologic treatment of bronchiolitis in infants and children: a systematic review. Arch Pediatr Adolesc Med 2004; 158: 127-37.

10. Leader S, Kohlhase K. Recent trends in severe respiratory syncytial virus (RSV) among US infants, 1997 to 2000. J Pediatr 2003; 143 (5 Suppl.): S127-32.

11. Mallory MD, Shay DK, Garrett J, Bordley WC. Bronchiolitis management preferences and the influence of pulse oximetry and respiratory rate on the decision to admit. Pediatrics 2003; 111: e45-51.

12. Deshpande SA, Northern V. The clinical and health economic burden of respiratory syncytial virus disease among children under 2 years of age in a defined geographical area. Arch Dis Child 2003; 88: 1065-9.

13. Tinsa F, Ben Rhouma A, Ghaffari H, et al. A randomized, controlled trial of nebulized terbutaline for the first acute bronchiolitis in infants less than 12-months-old. Tunis Med 2009; 87: 200-3.

14. Cheney J, Barber S, Altamirano L, et al. A clinical pathway for bronchiolitis is effective in reducing readmission rates. J Pediatr 2005; 147: 622-6.
15. Gadomski AM, Bhasale AL. Bronchodilators for bronchiolitis Cochrane Database Syst Rev 2006; 3: CD001266.

16. Kellner JD, Ohlsson A, Gadomski AM, Wang EE. Bronchodilators for bronchiolitis. Cochrane Database Syst Rev 2000; 2: CD001266.

17. American Academy of Pediatrics Subcommittee on Diagnosis and Management of Bronchiolitis. Diagnosis and management of bronchiolitis. Pediatrics 2006; 118: 1774-93.

18. Everard ML, Bara A, Kurian M, Elliott TM, Ducharme F, Mayowe $V$. Anticholinergic drugs for wheeze in children under the age of two years. Cochrane Database Syst Rev 2005; 3: CD001279.

19. Karadag B, Ceran O, Guven G, et al. Efficacy of salbutamol and ipratropium bromide in the management of scute bronchiolitis - A Clinical Trial. Respiration 2008; 76: 283-7.

20. Kellner JD, Ohlsson A, Gadomski AM, Wang EE. Bronchodilators for bronchiolitis. Cochrane Database Syst Rev 2000; 2: CD001266.

21. Hartling L, Wiebe N, Russell K, Patel H, Klassen TP. Epinephrine for bronchiolitis. Cochrane Database Syst Rev 2004; 1: CD003123.

22. Garrison MM, Christakis DA, Harvey E, Cummings P, Davis RL Systemic corticosteroids in infant bronchiolitis: A metaanalysis. Pediatrics 2000; 105: e44.

23. Patel H, Platt R, Lozano JM, Wang EE. Glucocorticoids for acute viral bronchiolitis in infants and young children. Cochrane Database Syst Rev 2004; 3: CD004878.

24. Teeratakulpisarn J, Limwattananon C, Tanupattarachai S, Limwattananon S, Teeratakulpisarn S, Kosalaraksa P. Efficacy of dexamethasone injection for acute bronchiolitis in hospitalized children: a randomized, double-blind, placebo-controlled trial. Pediatr Pulmonol 2007; 42: 433-9.

25. Mesquita M, Castro-Rodríguez JA, Heinichen L, Farina E, Iramain R. Single oral dose of dexamethasone in outpatients with bronchiolitis: a placebo controlled trial. Allergol Immunopathol (Madr) 2009; 37: 63-7.

26. Corneli HM, Zorc JJ, Mahajan P, et al. A multicenter, randomized, controlled trial of dexamethasone for bronchiolitis. N Engl J Med 2007; 357: 331-9. 